\title{
POLYNOMIALS FOR HYPEROVALS OF DESARGUESIAN PLANES
}

\section{CHRISTINE M. O'KEEFE and TIM PENTTILA}

(Received 27 October 1989; revised 2 March 1990)

Communicated by L. Caccetta

\begin{abstract}
This paper studies $o$-polynomials, that is, polynomials which represent hyperovals in Desarguesian projective planes of even order. We present theoretical restrictions on the form that $o$-polynomials can have, and we determine the number of $o$-polynomials corresponding to each of the known classes of hyperovals (other than Cherowitzo's). We use this to give the number of known $o$-polynomials for the fields of orders 4, 8, 16 and 32. Exploratory computer searches for 0 -polynomials for fields of small orders greater than 16 are reported.
\end{abstract}

1991 Mathematics subject classification (Amer. Math. Soc.) 51 E 20.

\section{Theoretical results}

Let $P G(2, q)$ be the projective plane over the field $G F(q)$ of order $q$, where $q$ is a power of a prime $p$. A hyperoval of $\operatorname{PG}(2, q)$ is a set of $q+2$ points, no three of which are collinear. An account of hyperovals appears in [5], but here we note a few relevant definitions and results. Firstly, hyperovals exist in $P G(2, q)$ if and only if $q$ is even. A hyperoval is regular if it contains $q+1$ points which are the points of a non-degenerate conic in $P G(2, q)$. Conversely, the points of a non-degenerate conic together with a unique further point called the nucleus of the conic provide an example of a hyperoval.

TheOREM 1.1 [5, 8.4.1]. If $q=2$ then every hyperoval is regular.

(C) 1991 Australian Mathematical Society 0263-6115/91 \$A2.00+0.00 
Proof. In $P G(2,2)$ a hyperoval consists of four points, any three of which form a conic.

By the transitivity of the collineation group $P \Gamma L(3, q)$ of $P G(2, q)$ on quadrangles, every hyperoval can be mapped by an element of $P \Gamma L(3, q)$ to one containing the fundamental quadrangle $(1,0,0),(0,1,0),(0,0,1)$ and $(1,1,1)$. A hyperoval which is the image under an element of $P \Gamma L(3, q)$ of a hyperoval $\mathscr{H}$ is equivalent to $\mathscr{H}$.

We therefore restrict our attention to $P G(2, q)$ with $q>2$ and consider only hyperovals which contain the fundamental quadrangle. The next result shows that these are related to permutation polynomials, that is, polynomials which are permutations when regarded as polynomial functions.

TheOREM $1.2[5,8.4 .2]$. A hyperoval $\mathscr{O}$ in $\operatorname{PG}(2, q)$ where $q>2$ is even can be written as

$$
\mathscr{D}(f)=\{(1, t, f(t)): t \in G F(q)\} \cup\{(0,1,0),(0,0,1)\}
$$

where $f$ is a permutation polynomial of degree at most $q-2$ satisfying $f(0)=0$ and $f(1)=1$. Further, for each $s \in G F(q)$, the polynomial $f^{(s)}$ where

is a permutation polynomial.

$$
f^{(s)}(x)=\frac{f(x+s)+f(s)}{x}, f^{(s)}(0)=0
$$

Permutation polynomials which arise in this way are called o-polynomials following Cherowitzo [1], and o-polynomials arising from equivalent hyperovals will be called equivalent. If $f$ is an o-polynomial then $f(0)=0$ and $f(1)=1$ imply that $f$ has no constant term and that the sum of the coefficients of $f$ is 1 . Other results concerning the terms appearing in an $o$-polynomial are the following three theorems.

THEOREM $1.3[14 ; 5,8.4 .2$ COROLLARY 1]. The coefficient of each term of odd power in an o-polynomial is zero.

For the next statement we need the following partial ordering $\preceq$ on the set of integers $n$ where $0 \leq n \leq q-1$. If

$$
b=\sum_{i=0}^{h-1} b_{i} 2^{i} \text { and } c=\sum_{i=0}^{h-1} c_{i} 2^{i}
$$

(where each $b_{i}$ and each $c_{i}$ is either 0 or 1 ) then $b \preceq c$ if and only if $b_{i} \leq c_{i}$ for all $i$. In other words, $b \preceq c$ if and only if all terms appearing in the binary expansion of $b$ also appear in the binary expansion of $c$. 
The main source of importance for this partial ordering is the following version of the binomial theorem in fields of characteristic 2 :

$$
(x+y)^{n}=\sum_{i \preceq n} x^{i} y^{n-i} .
$$

Another way of stating this is

$$
(x+y)^{\alpha_{1}+\alpha_{2}+\cdots+\alpha_{k}}=\prod_{j=1}^{k}\left(x^{\alpha_{j}}+y^{\alpha_{j}}\right),
$$

where $n=\alpha_{1}+\alpha_{2}+\cdots+\alpha_{k}$ and the $\alpha_{j}$ are distinct powers of 2 .

THeOREM 1.4 [3]. A polynomial $f$ of degree at most $q-2$ satisfying $f(0)=0$ and $f(1)=1$ is an o-polynomial if and only if the coefficient of $x^{c}$ in $f(x)^{b}\left(\bmod x^{q}-x\right)$ is zero for all pairs of integers $(b, c)$ satisfying $1 \leq b \leq c \leq q-1, b \neq q-1$ and $b \preceq c$.

Note that the case $b=1$ gives the result of Theorem 1.3. In addition, this condition can be analysed to give further equations relating the coefficients of $f$ as follows.

THEOREM 1.5. Let $f(x)=\sum_{i=1}^{q-2} a_{i} x^{i}$ and suppose that $\sum_{i=1}^{q-2} a_{i}=1$. Then $f$ is an o-polynomial if and only if for all $b$ with $1 \leq b \leq q-2$ and all $c$ with $b \preceq c$

$$
\sum \prod_{k=1}^{n} a_{i_{k}}^{\alpha_{k}}=0,
$$

where $b=\alpha_{1}+\alpha_{2}+\cdots+\alpha_{n}$, the $\alpha_{j}$ are distinct powers of 2 and the sum is over all $i_{k}$ with $c=\sum_{k=1}^{n} i_{k} \alpha_{k}(\bmod q-1)$.

Proof. The polynomial $f(x)^{b}$ is

$$
\left(\sum_{i=1}^{q-2} a_{i} x^{i}\right)^{\alpha_{1}+\alpha_{2}+\cdots+\alpha_{n}}=\prod_{j=1}^{n}\left(\sum_{i=1}^{q-2} a_{i}^{\alpha_{j}} x^{i \alpha_{j}}\right) .
$$

By Theorem 1.4, if $b \preceq c$ then the coefficient of $x^{c}$ in the right hand-side is zero; so

$$
\sum \prod_{k} a_{i_{k}}^{\alpha_{k}}=0
$$

When investigating $o$-polynomials, two coefficients can often be assumed 
to be equal. More precisely,

THEOREM 1.6. Let $f(x)=\sum_{k=1}^{(q-2) / 2} a_{2 k} x^{2 k}$ be an o-polynomial such that for some $i$ and $j$, with $i-j$ coprime to $q-1, a_{2 i} \neq 0$ and $a_{2 j} \neq 0$. Then $f$ is equivalent to an o-polynomial $g(x)=\sum_{k=1}^{(q-2) / 2} b_{2 k} x^{2 k}$ with $b_{2 i}=b_{2 j}$ and, for all $k, b_{2 k}=0$ if and only if $a_{2 k}=0$.

Proof. Let $f(x)=\sum_{k=1}^{(q-2) / 2} a_{2 k} x^{2 k}$ with $a_{2 i} \neq 0, a_{2 j} \neq 0$ and $(i-j, q-$ $1)=1$. The hyperoval $\mathscr{D}(f)$ is mapped by the homography with matrix

$$
\mathscr{K}_{s}=\left(\begin{array}{ccc}
1 & 0 & 0 \\
0 & s^{-1} & 0 \\
0 & 0 & f(s)^{-1}
\end{array}\right), \quad s \in G F(q)-\{0\}
$$

to a hyperoval $\mathscr{M}_{s}(\mathscr{D}(f))$ which still contains the fundamental quadrangle, since $\mathscr{M}_{s}$ fixes the points $(1,0,0),(0,1,0)$ and $(0,0,1)$ and maps the point $(1, s, f(s))$ to $(1,1,1)$. Thus the $o$-polynomial $f_{s}$ of $\mathscr{M}_{s}(\mathscr{D}(f))$ is equivalent to $f$. Since

$$
\left(\begin{array}{ccc}
1 & 0 & 0 \\
0 & s^{-1} & 0 \\
0 & 0 & f(s)^{-1}
\end{array}\right)\left(\begin{array}{c}
1 \\
t \\
f(t)
\end{array}\right)=\left(\begin{array}{c}
1 \\
s^{-1} t \\
f(s)^{-1} f(t)
\end{array}\right)=\left(\begin{array}{c}
1 \\
u \\
f(s)^{-1} f(s u)
\end{array}\right)
$$

this 0 -polynomial is

$$
f_{s}(x)=f(s)^{-1} f(s x)=\left(\sum_{k=1}^{(q-2) / 2} a_{2 k} s^{2 k}\right)^{-1} \sum_{k=1}^{(q-2) / 2} a_{2 k} s^{2 k} x^{2 k} .
$$

The ratio of the coefficient of $x^{2 i}$ to the coefficient of $x^{2 j}$ in $f_{s}$ is $s^{2(i-j)}\left(a_{2 i} / a_{2 j}\right)$ for $s \in G F(q)-\{0\}$. Since $(i-j, q-1)=1$, the ratio takes on $q-1$ distinct non-zero values in the $q-1 \quad o$-polynomials $f_{s}$ equivalent to $f$, and the result follows.

When conducting a computer search for $o$-polynomials and using this theorem, each $o$-polynomial with $a_{2 i}=a_{2 j}$, non-zero, gives rise to $q-1$ $o$-polynomials. The number of $o$-polynomials can be enough to characterize the hyperovals by using the ideas of Theorem 1.8 below.

Corollary. A binomial o-polynomial $f(x)=a x^{2 i}+b x^{2 j}$ has $(i-j, q-$ 1) $\neq 1$. In particular, if $q-1$ is a prime then there are no binomial $o$ polynomials representing hyperovals in $P G(2, q)$.

Proof. Suppose that $f(x)=a x^{2 i}+b x^{2 j}$ is a binomial $o$-polynomial for $P G(2, q)$ and that $(i-j, q-1)=1$. The theorem implies that $f$ is 
equivalent to the $o$-polynomial $f_{s}(x)=a x^{2 i}+a x^{2 j}$. But $f_{s}(1)=1$ means that $a+a=1$, a contradiction since when $q$ is even, $a+a=0$ for any $a \in G F(q)$.

For example, $q=8$ and $q=32$ have $q-1$ prime so hyperovals in $P G(2,8)$ or $P G(2,32)$ can never have a binomial $o$-polynomial.

An $o$-polynomial defined over a proper subfield has further restrictions, as remarked in [3].

THEOREM 1.7. If $f$ is an o-polynomial for $P G\left(2, q^{r}\right)$ with coefficients in $G F(q)$ then $f$ is congruent to an o-polynomial for $P G(2, q)$ modulo $x^{2 q}+x^{2}$.

Proof. If $f$ is considered to be a polynomial over $G F(q)$, then it is an $o$-polynomial for $\operatorname{PG}(2, q)$, since it defines a set of points, no three collinear, of the correct cardinality. It follows that $f \equiv g\left(\bmod x^{q}+x\right)$ for some $o$ polynomial $g$ for $P G(2, q)$. By Theorem 1.3 both $f$ and $g$ are squares, say $f=f_{1}^{2}$ and $g=g_{1}^{2}$. Now $f_{1}(a)^{2}=g_{1}(a)^{2}$ for all $a \in G F(q)$, so $f_{1}(a)=g_{1}(a)$ for all $a \in G F(q)$. Therefore $f_{1} \equiv g_{1}\left(\bmod x^{q}+x\right)$; so $f_{1}^{2} \equiv g_{1}^{2}\left(\bmod x^{2 q}+x^{2}\right)$. (In fact $f(x)=g(x)+r(x)^{2}\left(x^{2 q}+x^{2}\right)$ for some polynomial $r(x)$, since $f$ is a square.)

Another useful observation, due to Cherowitzo [1], is that if $\sum_{i=1}^{(q-1) / 2} a_{2 i} x^{2 i}$ is an $o$-polynomial then $\sum_{i=1}^{(q-1) / 2} a_{2 i}^{\alpha} x^{2 i}$ is an equivalent $o$-polynomial, where $\alpha$ is any automorphism of $G F(q)$.

These results can be used to conduct a computer search for $o$-polynomials, particularly in small fields. An exhaustive list of $o$-polynomials for a given value of $q$ constitutes a means of characterization of the hyperovals of $P G(2, q)$ for that value of $q$. For this purpose, it is useful to know how many $o$-polynomials are equivalent to a given $o$-polynomial. This depends on the particular hyperoval, as is shown in the next result.

THEOREM 1.8. Let $\mathscr{H}$ be a hyperoval of $P G(2, q)$ where $q=2^{h}$ and $h \geq 2$, containing the fundamental quadrangle $(1,0,0),(0,1,0),(0,0,1)$ and $(1,1,1)$. Let the o-polynomial of $\mathscr{H}$ be $f$ and let $G$ denote the stabiliser of $\mathscr{H}$ in $P \Gamma L(3, q)$. Then the number of o-polynomials equivalent to $f$ is

$$
\frac{h(q+2)(q+1) q(q-1)}{|G|}
$$


Proof. The $o$-polynomials equivalent to $f$ are precisely the $o$-polynomials associated with the hyperovals which are images under $P \Gamma L(3, q)$ of $\mathscr{H}$ and which contain the fundamental quadrangle. We find the number of such hyperovals by counting in two ways the pairs $\left(\mathscr{H}^{\prime}, Q\right)$ where $\mathscr{H}^{\prime}$ is an image under $P \Gamma L(3, q)$ of $\mathscr{H}$ and $Q$ is a quadrangle contained in $\mathscr{H}^{\prime}$. Letting $N$ denote the number of hyperovals containing a fixed quadrangle $Q$ which are images under $P \Gamma L(3, q)$ of $\mathscr{H}$, we obtain

$$
|P \Gamma L(3, q): G| \cdot\left(\begin{array}{c}
q+2 \\
4
\end{array}\right)=\frac{\left(q^{2}+q+1\right)\left(q^{2}+q\right) q^{2}(q-1)^{2}}{4 !} . N
$$

and the result follows.

Corollary. Table 1 displays, for each of the known hyperovals $\mathscr{H}$, the order $|G(\mathscr{H})|$ of the stabiliser of that hyperoval and hence the number $N(\mathscr{H})$ of equivalent o-polynomials representing that hyperoval. For the details, see [9]. The known hyperovals of $P G(2, q) . q=2^{h} \geq 4$, are the following.

(1) the regular hyperovals $\mathscr{R}=\mathscr{D}\left(x^{2}\right)$;

(2) the translation hyperovals $\mathscr{T}=\mathscr{D}\left(x^{2^{i}}\right)$, where $(i, h)=1,[12]$;

(3) the hyperoval $\mathscr{D}\left(x^{6}\right)$, where $h \geq 5$ is odd, $[13,14]$;

(4) the Lunelli-Sce hyperoval $\mathscr{L}=\mathscr{D}(f)$, where $f(x)=x^{12}+x^{10}+$ $\eta^{11} x^{8}+x^{6}+\eta^{2} x^{4}+\eta^{9} x^{2}, q=16$ and $\eta$ is a primitive root satisfying $\eta^{4}=\eta+1,[6]$

(5) the Glynn hyperovals $\mathscr{G}_{1}=\mathscr{D}\left(x^{3 \sigma+4}\right)$, where $h \geq 7$ is odd and $\sigma^{2} \equiv 2$ $(\bmod q-1),[2]:$

(6) the Glynn hyperovals $\mathscr{G}_{2}=\mathscr{D}\left(x^{\sigma+\lambda}\right)$, where $h \geq 7$ is odd, $\sigma^{2} \equiv 2$ $(\bmod q-1), \lambda^{4} \equiv 2(\bmod q-1)$ and $\lambda^{2} \equiv \sigma(\bmod q-1)$ [2];

(7) the Payne hyperovals $\mathscr{P}=\mathscr{D}\left(x^{1 / 6}+x^{3 / 6}+x^{5 / 6}\right)$, where $h \geq 5$ is odd and the exponents are read modulo $q-1,[10]$;

(8) the Cherowitzo hyperovals $\mathscr{C}=\mathscr{D}\left(x^{\sigma}+x^{\sigma+2}+x^{3 \sigma+4}\right)$, where $h=5,7$ or 9 and $\sigma^{2} \equiv 2(\bmod q-1),[1]$.

Let $\mathscr{H}$ be a hyperoval containing the fundamental quadrangle $Q$ and with $o$-polynomial $f$. The group of projectivities which stabilises $Q$ has order 24 , and any of its elements maps $\mathscr{H}$ to an equivalent hyperoval. Using this observation, Cherowitzo [1] gives a list of images of the point $(1, t, f(t))$ which implicitly define $24 o$-polynomials equivalent to $f$. In particular he finds the $o$-polynomials of Theorem 1.9.

THEOREM 1.9 [1]. The following polynomials $g$ are o-polynomials equivalent to a given o-polynomial $f$ : 
Table 1

\begin{tabular}{|l|l|l|}
\hline hyperoval & $|G(\mathscr{H})|$ & $N(\mathscr{H})$ \\
\hline regular $\mathscr{R}, q=2,4$ & $(q+2)(q+1) q(q-1) h$ & 1 \\
regular $\mathscr{R}, q \geq 8$ & $(q+1) q(q-1) h$ & $q+2$ \\
irregular translation $\mathscr{T}$ & $q(q-1) h$ & $(q+2)(q+1)$ \\
$\mathscr{D}\left(x^{6}\right), q=32$ & $3(q-1) h=465$ & $(q+2)(q+1) q / 3=11968$ \\
$\mathscr{D}\left(x^{6}\right), q \geq 128$ & $(q-1) h$ & $(q+2)(q+1) q$ \\
Lunelli-Sce $\mathscr{L}, q=16$ & $(q+2) 2 h=144$ & $(q+1) q(q-1) / 2=2040$ \\
Glynn $\mathscr{S}_{1}$ & $(q-1) h$ & $(q+2)(q+1) / q$ \\
Glynn $\mathscr{S}_{2}, q=128$ & $3(q-1) h=2667$ & $(q+2)(q+1) q / 3$ \\
Glynn $\mathscr{G}_{2}, q>128$ & $(q-1) h$ & $(q+2)(q+1) q$ \\
Payne $\mathscr{P}$ & $2 h$ & $(q+2)(q+1) q(q-1) / 2$ \\
Cherowitzo $\mathscr{C}, q=32$ & $h=5$ & $(q+2)(q+1) q(q-1)$ \\
Cherowitzo $\mathscr{C}, q \geq 128$ & divisible by $h$ & $\leq(q+2)(q+1) q(q-1)$ \\
\hline
\end{tabular}

(2) $g(x)=f^{-1}(x)$;

(5) $g(x)=x f(1 / x), g(0)=0$;

(6) $g(x)=f(x+1)+1$;

(11) $g(x)=(x+1) f(1 /(x+1))+1, g(1)=1$;

(12) $g(x)=x+x f(1+(1 / x)), g(0)=0$;

(16) $g(x)=x+(x+1) f(x /(x+1)), g(1)=1$;

as well as each of the above with $f$ replaced by $f^{-1}$. (Here the numbering follows that of [1, Result 2]).

If we now consider projectivities fixing $(1,0,0),(0,1,0)$ and $(0,0,1)$ pointwise but not necessarily fixing the point $(1,1,1)$ we obtain the following.

THEOREM 1.10. If $f$ is an o-polynomial then the following $q-1$ polynomials $f_{s}$ are o-polynomials equivalent to $f$ :

$$
f_{s}(x)=f(s)^{-1} f(s x), \text { for } s \in G F(q)-\{0\} .
$$

Proof. The homographies with matrix $\mathscr{M}_{s}, s \in G F(q)-\{0\}$, introduced in Theorem 1.6 map the hyperoval $\mathscr{D}(f)$ to equivalent hyperovals containing the fundamental quadrangle and hence give $q-1 \quad o$-polynomials $f_{s}$ equivalent to $f$ as in the statement of the theorem. 
THEOREM 1.11. When $q \geq 8$ the $q+2$ equivalent o-polynomials representing a regular hyperoval are exactly

(1) the 3 monomials $x^{2}, x^{1 / 2}$ and $x^{-1}$,

(2) the $q-1$ polynomials

$$
g_{s}(x)=\sum_{i=1}^{(q-2) / 2}(s+1) s^{2 i-1} x^{2 i}, \quad \text { for } s \in G F(q)-\{0\} .
$$

Proof. A regular hyperoval is represented by the $o$-polynomial $f(x)=$ $x^{2}$. The other two monomials listed in (1) are $o$-polynomials equivalent to $x^{2}$ by Theorem 1.9 (2) and (5). It can be verified that these three are distinct for $q \geq 8$. By Theorem 1.9 (11), the regular hyperoval is also represented by the $o$-polynomial

$$
g(x)=(x+1) f(1 /(x+1))+1=\frac{(x+1)}{(x+1)^{2}}+1=\frac{x}{x+1} \text { and } g(1)=1 .
$$

The o-polynomial $g$ can also be expressed as $g(x)=(x+1)^{q-2}+1=$ $\sum_{i=1}^{(q-2) / 2} x^{2 i}$. It now follows from Theorem 1.10 that $g$ is equivalent to the $o$-polynomials

$$
g_{s}(x)=\frac{s+1}{s} \sum_{i=1}^{(q-2) / 2}(s x)^{2 i}=\sum_{i=1}^{(q-2) / 2}(s+1) s^{2 i-1} x^{2 i} .
$$

By Corollary (2) to Theorem 1.8 there are $q+2 o$-polynomials equivalent to $f$, and the proof is complete.

\section{2. $O$-polynomials for $P G(2,4)$ and $P G(2,8)$}

THEOREM 2.1. In $P G(2,4)$ every hyperoval is regular. In particular, there is a unique o-polynomial $f(x)=x^{2}$ for $P G(2,4)$.

Proof. A hyperoval in $P G(2,4)$ has six points, any five of which uniquely determine a conic. By Theorems 1.2 and 1.3 , an $o$-polynomial for $P G(2,4)$ has only terms with even powers of $x$ appearing, has degree at most $q-2=2$ and has the sum of coefficients equal to 1 . Since $f(x)=x^{2}$ is an $o$ polynomial the result follows.

COROLLARY. The stabiliser of a regular hyperoval in $P G(2,4)$ has order 720 , is isomorphic to $S_{6}$ and acts naturally on the points of the hyperoval.

Proof. By Theorem 1.4 and (2) above, the stabiliser $G$ of a regular hyperoval $\mathscr{H}$ in $P G(2,4)$ has order $2(4+2)(4+1) 4(4-1)$. We have a 
homomorphism from $G$ into $S_{6}$ with kernel the pointwise stabiliser of $\mathscr{H}$, which is trivial. To see that it is onto, note that the orders of $G$ and $S_{6}$ are equal.

THEOREM 2.2. In $P G(2,8)$ every hyperoval is regular. The o-polynomials for $P G(2,8)$ are $a_{6} x^{6}+a_{4} x^{4}+a_{2} x^{2}$ where the values of $a_{6}, a_{4}, a_{2}$ are given in Table 2. $G F(8)$ has primitive root $\varepsilon$ where $\varepsilon^{3}=\varepsilon^{2}+1$, and so $\varepsilon^{7}=1$.

Table 2

\begin{tabular}{|c|cccccccccc|}
\hline$a_{6}$ & 0 & 0 & $\varepsilon^{3}$ & $\varepsilon^{3}$ & $\varepsilon^{5}$ & $\varepsilon^{5}$ & $\varepsilon^{6}$ & $\varepsilon^{6}$ & $\varepsilon^{7}$ & $\varepsilon^{7}$ \\
$a_{4}$ & 0 & $\varepsilon^{7}$ & $\varepsilon^{1}$ & $\varepsilon^{4}$ & $\varepsilon^{2}$ & $\varepsilon^{4}$ & $\varepsilon^{1}$ & $\varepsilon^{2}$ & 0 & $\varepsilon^{7}$ \\
$a_{2}$ & $\varepsilon^{7}$ & 0 & $\varepsilon^{6}$ & $\varepsilon^{5}$ & $\varepsilon^{6}$ & $\varepsilon^{3}$ & $\varepsilon^{3}$ & $\varepsilon^{5}$ & 0 & $\varepsilon^{7}$ \\
\hline
\end{tabular}

Proof. By Theorems 1.2 and 1.3, an $o$-polynomial for $P G(2,8)$ is of the form $a_{6} x^{6}+a_{4} x^{4}+a_{2} x^{2}$ where $a_{6}+a_{4}+a_{2}=1$. Consider the equations of Theorem 1.5 for the cases $b=3, c=3$ and $b=3, c=7$, which are

$$
a_{4}^{2} a_{2}+a_{2}^{2} a_{6}=0 \text { and } a_{6}^{2} a_{2}+a_{4}^{2} a_{6}=0
$$

respectively. There are 10 solutions $\left(a_{6}, a_{4}, a_{2}\right)$ with $a_{2}+a_{4}+a_{6}=1$ to this system of equations. This can be seen by noting that the system can be rewritten as $\left(a_{4}^{2}+a_{2} a_{6}\right) a_{2}=0$ and $\left(a_{4}^{2}+a_{2} a_{6}\right) a_{6}=0$ so that the solutions are the point $(0,1,0)$ together with the points of the conic $a_{4}^{2}=a_{2} a_{6}$. Since Theorem 1.11 constructs $10 o$-polynomials representing regular hyperovals, it follows that they are the only 0 -polynomials for $P G(2,8)$.

Note the curious fact mentioned by Glynn [3] that the triples $\left(a_{6}, a_{4}, a_{2}\right)$ with $a_{6} x^{6}+a_{4} x^{4}+a_{2} x^{2}$ an 0 -polynomial themselves form a regular hyperoval in $P G(2,8)$.

\section{O-polynomials for $P G(2,16)$}

By Theorems 1.2 and 1.3, an 0 -polynomial for $P G(2,16)$ has the form

$$
a_{14} x^{14}+a_{12} x^{12}+a_{10} x^{10}+a_{8} x^{8}+a_{6} x^{6}+a_{4} x^{4}+a_{2} x^{2}
$$

where $a_{i} \in G F(16)$ for all $i$ and $a_{14}+a_{12}+a_{10}+a_{6}+a_{4}+a_{2}=1$. There are $16^{6}=2^{24}$ such polynomials, that is, about 17 million. Theorem 1.2 provides 
an easily programmed test of whether or not a given polynomial is an $o$ polynomial. The output of a program which deals with all the polynomials of the above form is given in [8]. Only 2058 of the polynomials are $o$ polynomials. This verifies the following result of Hall [4], also obtained by computer.

THEOREM 3.1 [4]. In $P G(2,16)$ there are two equivalence classes of hyperovals, namely the regular hyperovals giving rise to 18 o-polynomials and the hyperovals first constructed by Lunelli and Sce [6] which give rise to 2040 o-polynomials.

COROLlary. The stabiliser of a Lunelli-Sce hyperoval in $P G(2,16)$ has order 144.

Proof. By Theorem 1.8, the stabiliser has order 4.18.17.16.15/2040.

In fact the stabiliser of the Lunelli-Sce hyperoval was first found in [11], without using Hall's result. This was used, together with Theorems 1.5 and 1.8 to prove Hall's result without a computer [7].

\section{4. $O$-polynomials for $P G(2,32)$}

The hyperovals in $P G(2,32)$ have not yet been classified, and as a consequence the number of $o$-polynomials for $P G(2,32)$ is unknown.

There are 5 known classes of hyperovals in $P G(2,32)$, giving a lower bound on the number of $o$-polynomials. The regular hyperovals provide 34 $o$-polynomials, the irregular translation hyperovals provide 1122 and the hyperoval $\mathscr{D}\left(x^{6}\right)$ gives rise to 11,968 . The Payne hyperovals provide 556,512 $o$-polynomials and the Cherowitzo hyperovals give rise to $1,113,024$.

The search space for $o$-polynomials for $P G(2,32)$ is at present too large for an exhaustive computer search to be justifiable. However, the following smaller searches have been made, with all $o$-polynomials found coming from one of the above 5 classes:

- polynomials with coefficients in $G F(2)([1,3])$;

- polynomials with one term ([2]).

These two searches were repeated, as well as

- polynomials with 2 to 4 terms;

- some polynomials with 5 terms.

5. Computer searches for hyperovals, $q>32$

The following spaces of polynomials over $G F(q)$ were searched for $o$ polynomials. In each case, any $o$-polynomials found correspond to a hyperoval belonging to one of the known classes. 
(1) $P G(2,64)$

- polynomials with coefficients in $G F(2)$ ([3]);

- some polynomials with coefficients in $G F(4)$ ([3]);

- polynomials with one term ([2]);

- polynomials with 2 to 3 terms.

(2) $P G(2,128)$

- polynomials with one term ([2]);

- polynomials with 3 terms and coefficients in $G F(2)$ ([1]).

(3) $P G(2,256)$

- polynomials with one term ([2]);

- 2040 o-polynomials for the Lunelli-Sce hyperoval with coefficients (from $G F(16)$ ) considered as elements of $G F(256)$.

(4) $P G(2,512)$

- polynomials with one term ([2]);

- polynomials with 3 terms and coefficients in $G F(2)$ whose exponents occur as monomial $o$-polynomials ([1]).

(5) $P G\left(2,2^{h}\right), h \leq 28$

- polynomials with one term $([2,3])$.

\section{Acknowledgement}

The first author acknowledges the support of an ARC Research Fellowship.

\section{References}

[1] W. Cherowitzo, 'Hyperovals in Desarguesian planes of even order', Ann. Discrete Math. 37 (1988), 87-94.

[2] D. G. Glynn, 'Two new sequences of ovals in finite Desarguesian planes of even order', Combinatorial Mathematics X, edited by L. R. A. Casse, pp. 217-229 (Lecture Notes in Mathematics 1036, Springer, 1983).

[3] _ 'A condition for the existence of ovals in $P G(2, q), q$ even', Geom. Dedicata, 32 (1989), 247-252.

[4] M. Hall, Jr., 'Ovals in the Desarguesian plane of order 16', Ann. Mat. Pura Appl. 102 (1975), 159-176.

[5] J. W. P. Hirschfeld, Projective Geometries over Finite Fields (Oxford University Press, 1979).

[6] L. Lunelli and M. Sce, ' $k$-archi completi nei piani proiettivi desarguesiani di rango $8 \mathrm{e}$ 16', Centro di Calcoli Numerici, Politecnico di Milano, (1958). 
[7] C. M. O'Keefe and T. Penttila, 'Hyperovals in $P G(2,16)$ ' European J. Combin., to appear.

[8] __, Polynomials Representing Hyperovals (University of Western Australia Research Report, June 1989/26).

[9]

[10] S. E. Payne, 'A new infinite family of generalized quadrangles', Congr. Numer. 49 (1985), 115-128.

[11] S. E. Payne and J. E. Conklin, 'An unusual generalized quadrangle of order sixteen', $J$. Combin. Theory Ser. $A 24$ (1978), 50-74.

[12] B. Segre, 'Sui $k$-archi nei piani finiti di caratteristica 2', Rev. Math. Pures Appl. 2 (1957), 289-300.

[13] _ ' 'Ovali e curve $\sigma$ nei piani di Galois di caratteristica due', Atti Accad. Naz. Lincei Rend. Cl. Sci. Fis. Mat. Natur. (8) 32 (1962), 785-790.

[14] B. Segre and U. Bartocci, 'Ovali ed altre curve nei piani di Galois di caratteristica due', Acta Arith. 8 (1971), 423-449.

The University of Western Australia

Nedlands, W.A. 6009

Australia 